\title{
Increasing physical activity levels in children: the ACTIVE project (ISRCTN75594310)
}

\section{Participant Flow}

A total of 13 schools were assessed for eligibility; four did not meet inclusion criteria of being deprived or located in one of Wales' most deprived areas. Two schools declined to participate. This meant 7 secondary schools took part in the RCT; four intervention and three control schools. Following initial school recruitment and head teacher approval, participants (in school year 9, aged 13 -14) were recruited for secondary outcome measures via school assemblies and information sheets. Consent was voluntary and involved both written parental consent and pupil assent forms. ACTIVE recruited 909 pupils. As well as this, members of the local council sport development team $(n=15)$ were recruited at twelve months to take part in a one-off focus group.

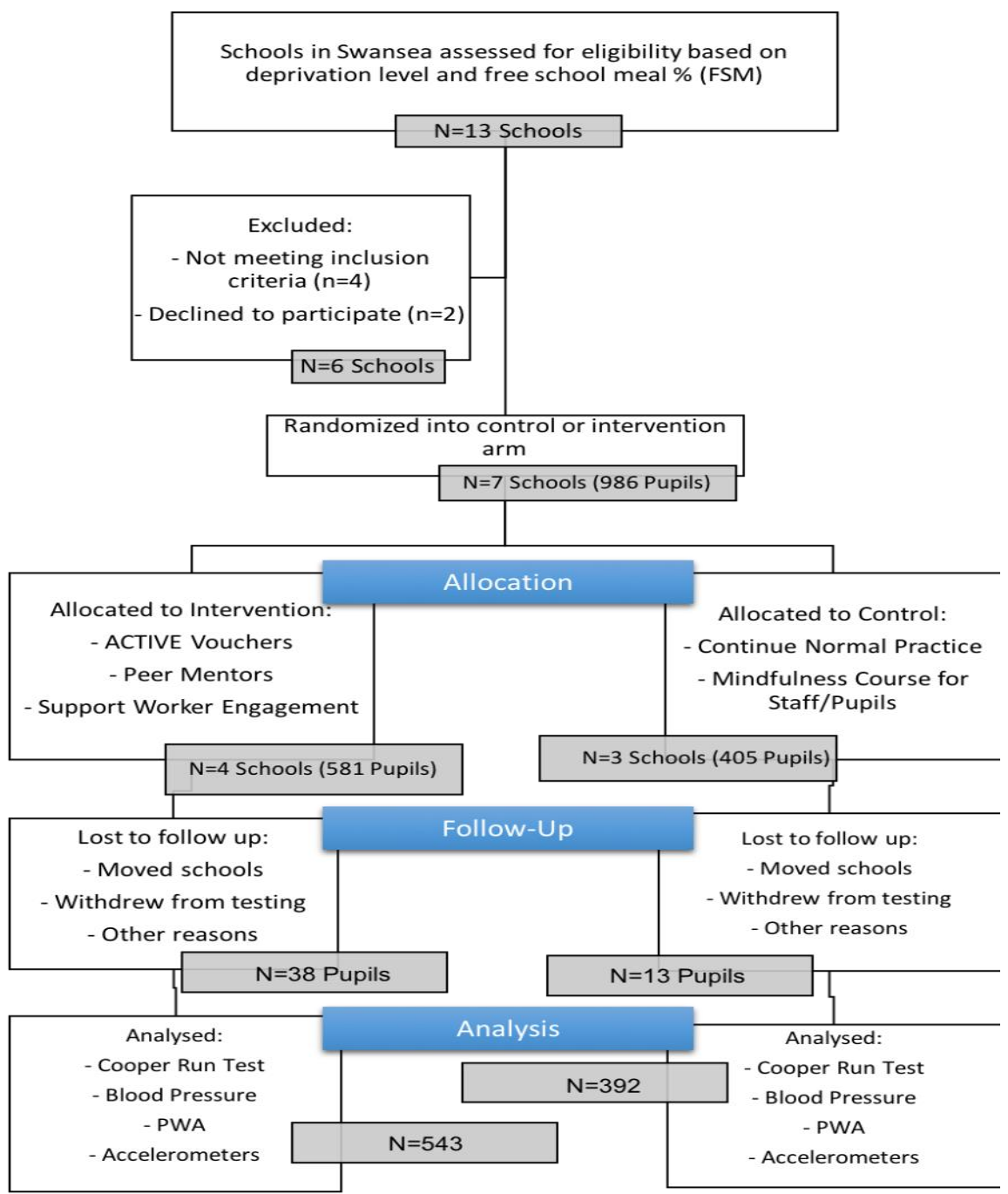




\section{Baseline Characteristics}

School level deprivation was derived from the Welsh Index of Multiple Deprivation (WIMD) which is used to identify areas of deprivation based on income, employment, health, education, access to services, community safety, environment and housing.

\begin{tabular}{lcc}
\hline \multicolumn{3}{l}{ Demographics of Schools } \\
\hline & $\begin{array}{c}\text { Number of pupils in } \\
\text { the study ( } n=\text { boys) }\end{array}$ & $\begin{array}{c}\text { Free school meal } \\
\% \text { in the school* }\end{array}$ \\
\hline School One & $113(n=56)$ & $26.4 \%$ \\
School Two & $231(n=107)$ & $19.2 \%$ \\
School Three & $125(n=59)$ & $10 \%$ \\
School Four & $128(n=62)$ & $38.1 \%$ \\
School Five & $97(n=50)$ & $50.5 \%$ \\
School Six & $142(n=77)$ & $21.7 \%$ \\
School Seven & $190(n=105)$ & $27.5 \%$ \\
*Free school meal eligibility is a marker that the family \\
income is below the poverty threshold
\end{tabular}

Randomisation into either intervention or control was done by an external statistician and occurred prior to baseline data collection. Characteristics of both arms are in the table. Due to the nature of the study, participants were aware

Characteristics of Intervention and Control Arm of which arm they were allocated.

\begin{tabular}{llll}
\hline Arm & Boys & Girls & Total \\
\hline Intervention & $254(48 \%)$ & $270(52 \%)$ & 524 \\
Control & $213(55 \%)$ & $172(45 \%)$ & 385 \\
\hline
\end{tabular}




\section{Outcome Measures}

Primary Outcome - Cardiovascular Fitness

Intervention Compared to Control

\begin{tabular}{|c|c|c|c|}
\hline Cooper Run (\% Fit) & Control $(n=384)$ & Intervention $(n=524)$ & Difference \\
\hline Baseline (Total) & $35.9 \%(n=138)$ & $33.5 \%(n=176)$ & $2.4 \%$ (95\% Cl: $-3.9 \%$ to $8.6 \%)$ \\
\hline 12 Months (Total) & $30.4 \%(n=117)$ & $33.0 \%(n=173)$ & $-2.6 \%$ (95\% Cl: $-8.6 \%$ to $3.6 \%)$ \\
\hline \multirow[t]{2}{*}{ Difference (Total) } & $5.4 \%$ (95\% Cl: $-.6 \%$ to $1.6 \%)$ & $0.5 \%$ (95\% Cl: $-4.5 \%$ to $5.7 \%)$ & $4.9 \%$ (95\% Cl: $2.7 \%$ to $7.6 \%)$ \\
\hline & Control Boys ( $n=212)$ & Intervention Boys ( $n=254)$ & Difference \\
\hline Baseline (Boys) & $22.1 \%(n=47)$ & $24.4 \%(n=62)$ & $-2.3 \%(95 \% \mathrm{Cl}:-9.9 \%$ to $5.5 \%)$ \\
\hline 12 Months (Boys) & $18.3 \%(n=39)$ & $20.0 \%(n=51)$ & $-1.7 \%(95 \% \mathrm{Cl}:-8.9 \%$ to $5.5 \%)$ \\
\hline \multirow[t]{2}{*}{ Difference (Boys) } & $3.8 \%$ (95\% Cl: $-3.5 \%$ to $11.1 \%)$ & $4.4 \%$ (95\% Cl: $-2.1 \%$ to $10.8 \%)$ & $-.6 \%(95 \% \mathrm{Cl}:-.2 \%$ to $4.6 \%)$ \\
\hline & Control Girls ( $n=172)$ & Intervention Girls $(n=270)$ & Difference \\
\hline Baseline (Girls) & $52.9 \%(n=91)$ & $42.2 \%(n=114)$ & $10.7 \%$ (95\% Cl: $11.5 \%$ to $20.2 \%)$ \\
\hline 12 Months (Girls) & $45.3 \%(n=78)$ & $45.1 \%(n=122)$ & $.2 \%(95 \% \mathrm{Cl}:-9.4 \%$ to $9.7 \%)$ \\
\hline \multirow[t]{2}{*}{ Difference (Girls) } & $7.5 \%$ (95\% Cl: $-2.8 \%$ to $17.9 \%)$ & $-2.9 \%$ (95\% Cl: $-10.8 \%$ to $4.8 \%)$ & $10.4 \%$ (95\% Cl: .4\% to $9.7 \%)$ \\
\hline & Control Deprived Pupils ( $n=146$ ) & Intervention Deprived Pupils ( $n=431$ ) & Difference \\
\hline Baseline (Deprived) & $36.3 \%(n=53)$ & $35.0 \%(n=151)$ & $1.3 \%$ (95\% Cl: $-7.7 \%$ to $10.2 \%)$ \\
\hline 12 Months (Deprived) & $30.8 \%(n=45)$ & $32.4 \%(n=140)$ & $-1.6 \%(95 \% \mathrm{Cl}:-10.4 \%$ to $7.1 \%)$ \\
\hline \multirow[t]{2}{*}{ Difference (Deprived) } & $5.5 \%$ (95\% Cl: $-4.4 \%$ to $15.4 \%)$ & $2.6 \%$ (95\% Cl: $-3.1 \% 8.2 \%)$ & $2.9 \%$ (95\% Cl: $-.4 \%$ to $7.9 \%)$ \\
\hline & Control Not Deprived Pupils ( $n=238)$ & Intervention Not Deprived Pupils ( $n=93$ ) & Difference \\
\hline Baseline (Not Deprived) & $35.7 \%(n=85)$ & $26.8 \%(n=25)$ & $8.9 \%$ (95\% Cl: $-2.4 \%$ to $20.1 \%)$ \\
\hline 12 Months (Not Deprived) & $30.2 \%(n=72)$ & $35.4 \%(n=33)$ & $-5.2 \%$ (95\% Cl: $-16.4 \%$ to $5.9 \%)$ \\
\hline Difference (Not Deprived) & $5.5 \%$ (95\% Cl: $-2.4 \%$ to $13.3 \%)$ & $-8.6 \%(95 \% \mathrm{Cl}:-20.6 \%$ to $3.4 \%)$ & $14.1 \%$ (95\% Cl: $4.1 \%$ to $19.2 \%)$ \\
\hline Cooper Run (Distance, $\mathrm{m}$ ) & Control (n=384) & Intervention $(n=524)$ & Difference \\
\hline Baseline (Total) & $1811.8( \pm 365.5)$ & $1781.9( \pm 373.5)$ & 29.9 (95\% $\mathrm{Cl}:-18.9$ to 78.6$)$ \\
\hline 12 Months (Total) & $1756.0( \pm 384.4)$ & $1762.3( \pm 421.1)$ & $-6.3(95 \% \mathrm{Cl}:-59.8$ to 47.2$)$ \\
\hline \multirow[t]{2}{*}{ Difference (Total) } & 55.7 (95\% Cl: 11.1 to 100.3$)$ & 19.6 (95\% Cl: -16.7 to 55.9$)$ & 36.1 (95\% Cl: -93.1 to 20.9$)$ \\
\hline & Control Boys $(n=212)$ & Intervention Boys $(n=254)$ & Difference \\
\hline Baseline (Boys) & $1989.9( \pm 346.0)$ & $2010.9( \pm 335.7)$ & $-21(95 \% \mathrm{Cl}:-83.1$ to 41.2$)$ \\
\hline 12 Months (Boys) & $1897.1( \pm 390.7)$ & $1953.2( \pm 400.3)$ & $-56.1(95 \% \mathrm{Cl}:-128.4$ to 16.3$)$ \\
\hline \multirow[t]{2}{*}{ Difference (Boys) } & $92.8(95 \% \mathrm{Cl}: 26.4$ to 159.1$)$ & 57.7 (95\% Cl: .2 to 115.2$)$ & 35.1 (95\% Cl: -122.2 to 52.0$)$ \\
\hline & Control Girls ( $n=172)$ & Intervention Girls ( $n=270)$ & Difference \\
\hline Baseline (Girls) & $1592.2( \pm 252.1)$ & $1566.5( \pm 263.1)$ & 25.7 (95\% $\mathrm{Cl}:-23.9$ to 75.3$)$ \\
\hline 12 Months (Girls) & $1582.1( \pm 295.8)$ & $1582.7( \pm 356.8)$ & $-.6(95 \% \mathrm{Cl}:-64.7$ to 63.5$)$ \\
\hline
\end{tabular}




\begin{tabular}{lll}
10.1 (95\% Cl: -46.6 to 66.9$)$ & $-16.1(95 \% \mathrm{Cl}:-61.4$ to 29.0$)$ & $26.2(95 \% \mathrm{Cl}:-98.5$ to 46.1$)$ \\
\hline Control Deprived Pupils $(n=146)$ & Intervention Deprived Pupils $(n=431)$ & Difference \\
\hline $1806.2( \pm 295.4)$ & $1783.1( \pm 371.6)$ & $23.1(95 \% \mathrm{Cl}:-43.4$ to 89.6$)$ \\
$1770.5( \pm 359.3)$ & $1763.5( \pm 412.5)$ & $7(95 \% \mathrm{Cl}:-68.2$ to 82.1$)$ \\
$35.7(95 \% \mathrm{Cl}:-27.9$ to 99.4$)$ & $19.6(95 \% \mathrm{Cl}:-19.2$ to 58.4$)$ & $16.1(95 \% \mathrm{Cl}:-92.2$ to 60.0$)$ \\
\hline Control Not Deprived Pupils $(n=238)$ & Intervention Not Deprived Pupils $(n=93)$ & Difference \\
\hline $1815.2( \pm 403.0)$ & $1776.3( \pm 384.1)$ & $38.9(95 \% \mathrm{Cl}:-56.7$ to 134.6$)$ \\
$1747.1( \pm 363.2)$ & $1756.5( \pm 461.1)$ & $-9.4(95 \% \mathrm{Cl}:-109.8$ to 91.1$)$ \\
$68.1(95 \% \mathrm{Cl}: 7.3$ to 128.7$)$ & $19.8(95 \% \mathrm{Cl}:-79.9$ to 119.5$)$ & $48.3(95 \% \mathrm{Cl}:-163.2$ to 66.6$)$
\end{tabular}

Baseline (Deprived)

12 Months (Deprived)

Difference (Deprived)

Baseline (Not Deprived)

12 Months (Not Deprived)

68.1 (95\% Cl: 7.3 to 128.7 )

19.8 (95\% Cl: -79.9 to 119.5$)$

48.3 (95\% Cl: -163.2 to 66.6$)$

Secondary Outcome - Cardiovascular Health

Intervention Compared to Control

Blood Pressure (\% High)

Baseline (Total)

12 Months (Total)

Difference (Total)

Baseline (Boys)

12 Months (Boys)

Difference (Boys)

Baseline (Girls)

12 Months (Girls)

Difference (Girls)

Baseline (Deprived)

12 Months (Deprived)

Difference (Deprived)

Baseline (Not Deprived)

12 Months (Not Deprived)

Difference (Not Deprived)

Control $(n=384)$

Intervention ( $n=524)$

$1.6 \%(n=6)$

$5.3 \%(n=28)$

Difference

$3.1 \%(n=12)$

$2.7 \%(n=14)$

$-3.7 \%$ (95\% Cl: $-5.5 \%$ to . $2 \%)$

$-1.4 \%$ (95\% Cl: $-3.7 \%$ to .6\%)

$2.6 \%$ (95\% Cl: $-3.0 \% 5.0 \%)$

Control Boys ( $n=212)$

$2.4 \%(n=5)$

$4.2 \%(n=9)$

Intervention Boys $(n=254)$

$.4 \%$ (95\% Cl: $-1.7 \%$ to $2.9 \%$ )

$-1.8 \%(95 \% \mathrm{Cl}:-1.7 \%$ to $5.7 \%) \quad 3.2 \%(95 \% \mathrm{Cl}:-.7 \%$ to $7.2 \%)$

Difference

$-4.3 \%$ (95\% Cl: .4\% to $8.3 \%)$

Control Girls ( $n=172)$

$.7 \%$ (95\% Cl: $-2.9 \%$ to $4.3 \%)$

Intervention Girls $(n=270) \quad$ Difference

$0.6 \%(n=1) \quad 4.1 \%(n=11)$

$1.7 \%(n=3) \quad 1.9 \%(n=5)$

$-3.5 \%$ (95\% Cl: .3\% to $6.6 \%)$

$-1.1 \%$ (95\% Cl: $-1.7 \%$ to $4.4 \%)$

$2.2 \%$ (95\% Cl: $-.7 \%$ to $5.4 \%)$

$.2 \%(95 \% \mathrm{Cl}:-3.3 \%$ to $2.7 \%)$

Control Deprived Pupils $(n=146)$

Intervention Deprived Pupils ( $n=431$ )

$-3.3 \%(95 \% \mathrm{Cl}:-2.1 \%$ to $3.7 \%)$

$2.0 \%(n=3)$

$3.4 \%(n=5) \quad 2.3 \%(n=10)$

$4.6 \%(n=20)$

Difference

$-2.6 \%$ (95\% Cl: $-1.5 \%$ to $5.3 \%$ )

$-1.4 \%(95 \% \mathrm{Cl}:-2.9 \%$ to $5.9 \%)$

$2.3 \%$ (95\% Cl: $-.1 \%$ to $4.9 \%)$

$1.1 \%$ (95\% Cl: $-1.6 \%$ to $5.5 \%$ )

Control Not Deprived Pupils ( $n=238$ )

Intervention Not Deprived Pupils ( $n=93$ )

$1.3 \%(n=3)$

$2.9 \%(n=7)$

$-3.7 \%(95 \% \mathrm{Cl}:-2.6 \%$ to $3.0 \%$ )

Augmentation Pressure $(\mathrm{mmHg})$

$-1.6 \%(95 \% \mathrm{Cl}:-1.1 \%$ to $4.8 \%)$

ifference

7.3\% (95\% Cl: $2.5 \%$ to $14.8 \%$ )

Control $(n=384)$

$4.3 \%$ (95\% Cl: $-3.2 \%$ to $12.2 \%)$

$-1.4 \%$ (95\% Cl: $-2.6 \%$ to $7.7 \%$ )

(PWA)

Intervention ( $n=524)$

Difference 


\begin{tabular}{|c|c|c|c|}
\hline Baseline (Total) & $4.9( \pm 2.5)$ & $5.0( \pm 2.6)$ & $-.1(95 \% \mathrm{Cl}:-.5$ to .1$)$ \\
\hline 12 Months (Total) & $4.1( \pm 2.2)$ & $4.0( \pm 2.4)$ & .1 (95\% Cl: -.2 to .3) \\
\hline \multirow{2}{*}{ Difference (Total) } & $.8(95 \% \mathrm{Cl}: .4$ to 1.1$)$ & 1 (95\% Cl: .7 to 1.3$)$ & $.2(95 \% \mathrm{Cl}:-.1$ to .7$)$ \\
\hline & Control Boys $(n=212)$ & Intervention Boys ( $n=254)$ & Difference \\
\hline Baseline (Boys) & $4.6( \pm 2.7)$ & $4.6( \pm 2.6)$ & .0 (95\% $\mathrm{Cl}:-.4$ to .5$)$ \\
\hline 12 Months (Boys) & $4.1( \pm 2.2)$ & $4.2( \pm 2.5)$ & $.1(95 \% \mathrm{Cl}:-.4$ to .4$)$ \\
\hline \multirow[t]{2}{*}{ Difference (Boys) } & $.5(95 \% \mathrm{Cl}:-.0$ to .9$)$ & $.4(95 \% \mathrm{Cl}:-.0$ to .8$)$ & $.1(95 \% \mathrm{Cl}:-.7$ to .5$)$ \\
\hline & Control Girls ( $n=172)$ & Intervention Girls ( $n=270)$ & Difference \\
\hline Baseline (Girls) & $5.2( \pm 2.3)$ & $5.5( \pm 2.4)$ & $-.3(95 \% \mathrm{Cl}:-.7$ to .1$)$ \\
\hline 12 Months (Girls) & $4.0( \pm 2.2)$ & $3.9( \pm 2.2)$ & $.1(95 \% \mathrm{Cl}:-.3$ to .5$)$ \\
\hline \multirow[t]{2}{*}{ Difference (Girls) } & 1.2 (95\% Cl: .7 to 1.7$)$ & $1.6(95 \% \mathrm{Cl}: 1.2$ to 2.0$)$ & $.1(95 \% \mathrm{Cl}:-.1$ to .9$)$ \\
\hline & Control Deprived Pupils ( $n=146$ ) & Intervention Deprived Pupils ( $n=431)$ & Difference \\
\hline Baseline (Deprived) & $4.5( \pm 3.2)$ & $5.2( \pm 2.4)$ & -.7 (95\% Cl: -1.2 to -.1$)$ \\
\hline 12 Months (Deprived) & $4.0( \pm 2.0)$ & $4.1( \pm 2.3)$ & $-1(95 \% \mathrm{Cl}:-.5$ to .3$)$ \\
\hline \multirow[t]{2}{*}{ Difference (Deprived) } & .5 (95\% Cl: -.1 to 1.1$)$ & 1.3 (95\% $\mathrm{Cl}: .8$ to 1.4$)$ & .8 (95\% Cl: .1 to 1.4$)$ \\
\hline & Control Not Deprived Pupils ( $n=238)$ & Intervention Not Deprived Pupils ( $n=93$ ) & Difference \\
\hline Baseline (Not Deprived) & $5.1( \pm 2.0)$ & $4.2( \pm 2.9)$ & .9 (95\% Cl: . 3 to 1.4$)$ \\
\hline 12 Months (Not Deprived) & $4.1( \pm 2.3)$ & $3.6( \pm 2.6)$ & .5 (95\% Cl: -.0 to 1.0$)$ \\
\hline Difference (Not Deprived) & 1.0 (95\% Cl: .5 to 1.3$)$ & .6 (95\% Cl: -.2 to 1.3$)$ & .4 (95\% Cl: -1.2 to .4$)$ \\
\hline Augmentation Index (\%) (PWA) & Control $(n=384)$ & Intervention $(n=524)$ & Difference \\
\hline Baseline (Total) & $9.5( \pm 4.0)$ & $10.0( \pm 4.6)$ & -.5 (95\% Cl: -1.1 to .0$)$ \\
\hline 12 Months (Total) & $7.4( \pm 3.2)$ & $7.6( \pm 4.3)$ & $-.2(95 \% \mathrm{Cl}:-.6$ to .3$)$ \\
\hline \multirow[t]{2}{*}{ Difference (Total) } & 2.1 (95\% Cl: 1.5 to 2.5$)$ & 2.4 (95\% Cl: 1.8 to 2.9$)$ & -.3 (95\% Cl: -.4 to 1.0$)$ \\
\hline & Control Boys $(n=212)$ & Intervention Boys ( $n=254)$ & Difference \\
\hline Baseline (Boys) & $8.8( \pm 3.9)$ & $9.1( \pm 4.8)$ & $-.3(95 \% \mathrm{Cl}:-1.1$ to .5$)$ \\
\hline 12 Months (Boys) & $7.9( \pm 3.1)$ & $8.1( \pm 4.5)$ & $-.2(95 \% \mathrm{Cl}:-.9$ to .5$)$ \\
\hline \multirow[t]{2}{*}{ Difference (Boys) } & $.9(95 \% \mathrm{Cl}: .1$ to 1.5$)$ & 1.0 (95\% Cl: .2 to 1.7$)$ & $-.1(95 \% \mathrm{Cl}:-.9$ to 1.1$)$ \\
\hline & Control Girls (n=172) & Intervention Girls ( $n=270)$ & Difference \\
\hline Baseline (Girls) & $10.3( \pm 3.9)$ & $10.9( \pm 4.1)$ & -.6 (95\% Cl: -1.3 to .2$)$ \\
\hline 12 Months (Girls) & $6.9( \pm 3.2)$ & $7.2( \pm 4.0)$ & -.3 (95\% Cl: -1.0 to .4$)$ \\
\hline \multirow[t]{2}{*}{ Difference (Girls) } & 3.4 (95\% Cl: 2.6 to 4.2$)$ & 3.7 (95\% Cl: 3.0 to 4.4$)$ & -.3 (95\% Cl: -.7 to 1.3$)$ \\
\hline & Control Deprived Pupils ( $n=146$ ) & Intervention Deprived Pupils ( $n=431)$ & Difference \\
\hline Baseline (Deprived) & $8.8( \pm 4.5)$ & $10.4( \pm 4.3)$ & $-1.6(95 \% \mathrm{Cl}:-2.4$ to -.8$)$ \\
\hline 12 Months (Deprived) & $7.6( \pm 3.0)$ & $7.8( \pm 3.9)$ & $-.2(95 \% \mathrm{Cl}:-.8$ to .5$)$ \\
\hline Difference (Deprived) & $1.2(95 \% \mathrm{Cl}: .2$ to 2.1$)$ & 2.6 (95\% Cl: 2.0 to 3.1$)$ & $-1.4(95 \% \mathrm{Cl}:-2.5$ to -.4$)$ \\
\hline
\end{tabular}


Baseline (Not Deprived)

12 Months (Not Deprived)

Difference (Not Deprived)

\section{Secondary Outcome - Exercise Motivation}

\section{Intervention Compared to Control}

Motivation (\% Autonomous)

Baseline (Total)

12 Months (Total)

Difference (Total)

Baseline (Boys)

12 Months (Boys)

Difference (Boys)

Baseline (Girls)

12 Months (Girls)

Difference (Girls)

Baseline (Deprived)

12 Months (Deprived)

Difference (Deprived)

Baseline (Not Deprived)

12 Months (Not Deprived)

Difference (Not Deprived)

\begin{tabular}{lll}
\hline Control Not Deprived Pupils $(n=238)$ & Intervention Not Deprived Pupils $(n=93)$ & Difference \\
\hline $9.9( \pm 3.6)$ & $8.3( \pm 5.2)$ & $\mathbf{1 . 6}(\mathbf{9 5 \%} \mathrm{Cl}: .6$ to 2.6$)$ \\
$7.4( \pm 3.3)$ & $7.0( \pm 5.5)$ & $.4(95 \% \mathrm{Cl}:-.5$ to 1.3$)$ \\
$2.5(95 \% \mathrm{Cl}: 1.9$ to 3.2$)$ & $1.3(95 \% \mathrm{Cl}:-.2$ to 2.8$)$ & $1.2(95 \% \mathrm{Cl}:-.1$ to 2.5$)$ \\
\hline
\end{tabular}

$1.2(95 \% \mathrm{Cl}:-1$ to 2.5$)$

$1.3(95 \% \mathrm{Cl} \cdot-.2$ to 2.8$)$

Control $(n=384) \quad$ Intervention $(n=524)$

Difference

$98.1 \%(n=378) \quad 97.1 \%(n=509) \quad 1 \%(95 \% \mathrm{Cl}:-.9 \%$ to $3 \%)$

$97.9 \%(n=377) \quad 97.9 \%(n=513) \quad 0 \%(95 \% \mathrm{Cl}:-1.8 \%$ to $1.9 \%)$

$.2 \%(95 \% \mathrm{Cl}:-1.5 \%$ to $2.1 \%) \quad-.8 \%(95 \% \mathrm{Cl}:-2.4 \%$ to $.9 \%) \quad .9 \%(95 \% \mathrm{Cl}:-.7 \%$ to $1.7 \%)$

Control Boys $(n=212)$

Intervention Boys ( $n=254) \quad$ Difference

98.1\% ( $n=209) \quad 97.6 \%(n=248) \quad .5 \%(95 \% \mathrm{Cl}:-2.1 \%$ to $3.1 \%)$

98.5\% $(n=210) \quad 97.6 \%(n=248) \quad .9 \%(95 \% \mathrm{Cl}:-1.5 \%$ to $3.4 \%)$

$-.4 \%$ (95\% Cl: $-2.9 \%$ to $1.9 \%) \quad 0 \%(95 \% \mathrm{Cl}:-2.1 \%$ to $2.1 \%) \quad-.4 \%(95 \% \mathrm{Cl}:-1.0 \% 2.6 \%)$

\begin{tabular}{lll}
\hline Control Girls $(n=172)$ & Intervention Girls $(n=270)$ & Difference \\
\hline $98.2 \%(n=169)$ & $96.6 \%(n=261)$ & $.6 \%(95 \% \mathrm{Cl}:-1.5 \%$ to $4.7 \%)$
\end{tabular}

$97.0 \%(n=167) \quad 98.1 \%(n=265) \quad-1.1 \%(95 \% \mathrm{Cl}:-3.9 \%$ to $1.8 \%)$

$1.2 \%(95 \% \mathrm{Cl}:-1.6 \%$ to $3.9 \%) \quad-1.5 \%(95 \% \mathrm{Cl}:-4.0 \%$ to $1.0 \%)$

$2.7 \%$ (95\% Cl: $-3.2 \%$ to $3.0 \%)$

Control Deprived Pupils ( $n=146)$

Intervention Deprived Pupils ( $n=431$ )

Difference

$97.2 \%(n=143) \quad 98.1 \%(n=423) \quad-0.9 \%(95 \% \mathrm{Cl}:-3.5 \%$ to $1.8 \%)$

99.3\% ( $\mathrm{n}=146) \quad 98.1 \%(\mathrm{n}=423) \quad 1.2 \%(95 \% \mathrm{Cl}:-1.1 \%$ to $3.5 \%)$

$-2.1 \%(95 \% \mathrm{Cl}:-5.0 \%$ to $.9 \%) \quad 0 \%(95 \% \mathrm{Cl}:-1.5 \%$ to $1.5 \%) \quad \mathbf{2 . 1 \%}(\mathbf{9 5} \% \mathrm{Cl}: . \mathbf{4} \%$ to $\mathbf{5 . 8 \%})$

Control Not Deprived Pupils ( $n=238) \quad$ Intervention Not Deprived Pupils $(n=93)$

$98.7 \%(n=235)$

$92.4 \%(n=86)$

$97.0 \%(n=231)$

$96.7 \%(n=90)$

Difference

$1.7 \%(95 \% \mathrm{Cl}:-.6 \%$ to $4.0 \%) \quad-4.3 \%(95 \% \mathrm{Cl}:-10.3 \%$ to $17.0 \%)$

$6.3 \%$ (95\% Cl: $2.1 \%$ to $10.3 \%$ )

$3 \%$ (95\% Cl: $-3.8 \%$ to $4.4 \%$ )

$6 \%(95 \% \mathrm{Cl}:-1.0 \%$ to $8.9 \%)$ 
Adverse Effects

There were no adverse events associated with this trial. 